\title{
Index to genera and species, Volume 10 (1999)
}

A

"Anoxus" boops 248

Acropiesta 187

Actaea spicata 138

Adelphocoris lineolatus (Goeze) 220

Ademon Haliday 68

Ademon decrescens (Nees) 68

- mutuator (Nees) 68

Adontopius Fischer 139

Adrastus dolini Wellschmied 118

Aegopodium podagraria L. 79, 82, 83, 86, 203

Aellenia subaphylla 228

Aeoloides heydeni (Reitter) 118

Aeshna cyanea 127

- juncea 127

Agnopius Fischer 81

Agramma minutum Horváth 234

- atricapillum (Spinola) 234

- laetum (Fallén) 234

Agromyza 69, 72, 79, 82, 86, 87, 145, 147

- ?pseudoreptans Nowakowski $=$ urticae Nowak 87

- abiens Zetterstedt 138, 143

- albitarsis Meigen 72, 74, 76, 82, 86, 87, 131, 136, 144

— alnibetulae Hendel 72, 138

— ambigua Fallén = niveipennis Zetterstedt 88, 131

- anthracina Meigen 83, 86, 138

— apfelbecki Strobl 136

- arunci Hering 138

- baetica Griffiths 72

- bicophaga Hering 79, 88

- ferruginosa Wulp 143

- frontella Rondani 74, 140

— genistae Hendel 79, 81, 83

- graminicola Hendel 131

- hendeli Griffiths 72, 130

- johannae de Meijere 145

- lathyri Hendel 88, 144

- lucida Hendel 72, 82, 131

- lyophaga Hering 76
- megalopsis Hering 69

— mobilis Meigen 73

- myosotidis Kaltenbach 143

- nigrella Rondani 74, 87, 88, 131, 152

— nigrescens Hendel 86, 132, 134

- nigriceps Hendel 88

- nigripes Meigen 69, 75, 82, 138

— nigrociliata Hendel 137

_ phragmitidis Hendel 72, 82, 131, 136

- potentillae (Kaltenbach)

$=$ spiraeae $(\mathrm{Kal}) 87,88,131$

- prespana Spencer 146

- pseudoreptans Nowakowski = urticae Nowako 86

- pseudorufipes Nowakowski 143

- reptans Fallén 72, 86, 131, 138, 143

- rufipes Meigen 73, 76, 143

- spenceri Griffiths 72, 134

- spiraeae Kaltenbach 81,83

- urticae Nowakowski 138

Agropyron repens (L.) 82

Agrostis 15

- canina 82, 138

Alhagi 230

Alisma plantago-aquatica 68

Allantus tricinctus 202

Alloeomimus kurdus Hoberlandt 223

Allophlebus Fischer 137

Allotypus Foerster 84

- irregularis 84

Alnus incana 138

- rotundifolia 72

Alopecosa fabrilis (Clerck) 6

Alopecurus pratensis 145

Amaranthus blitum 132, 134

Amauromyza (Trilobomyza) flavifrons (Meigen) 80,136

- (T.) gyrans (Fallén) 79, 81

- (T.) labiatarum (Hendel) 83, 87, 134, 145

- (T.) verbasci (Bouché) 134, 136, 137, 145

- lamii Kaltenbach 145

Amblyscelio 187 
Amblytylus concolor Jakovlev 226

- peitho Linnavuori 226

Ampedus sinuatus Germar 118

Amygdalus lycioides 222

Anabasis 223

Anacaena globulus (Paykull) 2

Anapus kirschbaumi Stål 222

Anax imperator 127

Ancistrocerus oviventris 194

- scoticus 194

Andrena 4

— lapponica Zetterstedt 4

Anemone sylvestris 134

Angelica archangelica ssp. litoralis (Fr.) Thell. 203

— silvestris 176

- sylvestris L. 79, 83, 86, 134, 138, 176, 203

Anoecia vagans (Koch) 94

Anonychiella alhagicola alhagicola

(Drapolyuk) 198230

- brevicornis (Reuter) 230

Anoxus 248

Anthemis tinctoria 134

Antherophagus nigricornis (F.) 50

Anthomyia 134

Anthrenus 241, 245

- museorum (Linnaeus) 241, 245

Anthriscus sylvestris 138

Anthyllis vulneraria 132, 134

Aphaenophyes richteri (Wagner) 230

Aphis chloris Koch 240

- craccivora Koch 240

- gossypii Glov. 240

- spiraecola Patch.(= citricola v.d.Goot) 240

Aphodius subterraneus (Linnaeus) 4

Aphria longilingua Rondani 1

- longirostris 1

Apodesmia Foerster 70

- taeniata Foerster 70

Aptesis albulatoria (Gravenhorst) 175, 176

Aquilegia 138

Arctium 83, 86, 132

Arrhenatherum elatius (L.) 82, 86, 137

Artemisia 106, 220, 221, 222, 224, 225, 229

— vulgaris L. 76, 77, 145

Aruncus vulgaris 138

Asperula odorata 87

Asphondylia verbasci Vallot. 133

Aster 106
- acris 106

- bellidiastrum 82

- linosyris 103, 106

Astragalus 222, 224, 228

Astrantia major 82,83

Atomaria 2

- lapponica Johnson 2

- pulchra Erichson 2

- rubricollis Bris. 50

- strandi Johnson 2

Atomophora pentheus Linnavuori 228

- astraia Linnavuori 228

- atripes Linnavuori 228

- maculosa maculosa Reuter 228

Atomorus victus 138

Atomoscelis onusta (Fieber) 224

Atoreuteus Foerster 152

- striatus Foerster 152

Atractodes romani Jussila 175, 176

- vicinus Foerster 176

Atriplex hortensis 71

Auchenocrepis alboscutellata Puton 229

- reuteri Jakovlev 229

Aulonotus Ashmead 69

\section{B}

Badezorus annulicornis (Reuter) 224

- ferdowsii Linnavuori 224

- immaculatus Linnavuori 224

- tomentosus (Reuter) 224

Ballota nigra 83,87

Bathystomus Foerster 152

- xanthopus Foerster 152

Bellis 83, 88

- perennis 152

- silvestris 83

- sylvestris 82

Berberis 76

Berytinus (Berytinus) hirticornis (Brullé) 234

- (Licinus) distinguendus (Ferrari) 234

Beta vulgaris $66,71,73,147,149,150,152$

Betarmon anatolicus Candèze 117

Bethylus 248

- boops (Thomson) 247, 248

Betula 244

Bibio lepidus Loew 115

- varipes Meigen 115

Bidens pilosa 240 
Biosteres Foerster 147, 150

- (Biosteres) brevisulcus (Thomson) 150

- (B.) carbonarius (Nees) 149, 150

- (B.) caudatulus (Thomson) 150, 151

-(B.) colorativentris (Fischer) 67, 151

- (B.) impressus 150

- (B.) magnicornis (Wesmael) 67, 147, 151

- (B.) placidus (Haliday) 151

- (B.) placodus 151

- (B.) spinaciae 152

- (B.) spinaciae (Thomson) 152

- (Chilotrichia) bicolor (Wesmael) 129, 147, 151

- (C.) blandus (Haliday) 147

— (C.) borealis (Zetterstedt) 147

- (C.) brevipalpis 147

- (C.) clypealis (Thomson) 147

- (C.) haemorrhoeus (Haliday) 148

-(C.) punctiscuta (Thomson) 67, 148

- (C.) rusticus (Haliday) 148

- (C.) scabriculus (Wesmael) 148, 149

- (C.) sylvaticus (Haliday) 129, 149

- (C.) ultor (Foerster) 149

- (C.) wesmaelii (Haliday) 149

- (Zetetes) haemorrhoeus 148

- (Zetetets) sylvaticus 149

- aureliae 78

— castaneiventris auct., Hellén 147

- indotatus Viereck 152

- longicauda 151

- melanocerus 152

- procerus 150

Bisnius cephalotes (Grav.) 50

Blepharidopterus diaphanus (Kirschbaum) 223

Boopidocoris nahidae Linnavuori 224

Brachynotocoris puncticornis Reuter 222

Brachypodium sylvaticum 136

Bracon borealis Zetterstedt 147

- carbonarius Nees 147, 149, 150

- circulator Nees 87

- decrescens Nees 68

- mutuator Nees ab Esenbeck 68

- nitidulator Nees 71

- pygmaeator Nees 131, 135

Bromus asper 87

Bryoporus rugipennis (Pand.) 50

Bunium creticum 134

Buvatina 249, 250
- iremella sp. n. 249, 250

- obscurella 250

- stroemella 250

- tineiformis Leraut 249, 250

C

Cacopsylla 201

Caenoscelis subdeplanata Bris. 50

Calais Castelnau 117

- brandti 118, 123, 124

- persicus Chassain 118

Calamagrostis 83

- arundinacea 82, 86, 136

- canescens 82, 136

- epigeios (L.) 82, 136

Caliroa algeriensis 185

- angustata Forsius 185

- carinata Zombori 185

- crypta 183, 184

- evodiae Xiao 185

- negativa Malaise 185

- toonae Li \& Guo 185

- tremulae Chevin 183, 185

- varipes (Klug) 183

- cerasi 185

Calligonum 228, 229

Callilepis nocturna (Linnaeus) 6

Calliphora vomitoria (Linnaeus) 71

Caltha palustris 132

Campanula 141

- rotundifolia 83

- scheuchzeri 83

- trachelium 81

Camponotus 223

Campoplex 241

- difformis 245

Camptotylidea albovittata (Reuter) 228

- flavescens (Puchkov) 228

- lineata (Reuter) 228

- persica Wagner 228

- suturalis (Reuter) 228

Camptotylus gracilis Wagner 218, 230

- meyeri Frey-Gessner 230

- reuteri Jakovlev 230

Camptozorus linnavuorii Kerzhner 224

Campylomma diversicorne Reuter 224

- lindbergi Hoberlandt 224

- lindbergi Hoberlandt 225

- nigronasuta Reuter 224, 225 
- simillima Jakovlev 224, 225

- verbasci (Meyer-Dür) 224

Campylosteira heissi Péricart 233

Capitophorus 201

Capparimyia savastanii (Martelli) 144

Capsicum anum conoides 240

Cardiophorus Eschscholtz 117

- alienus Dolin 121

- arnoldii Dolin 125

- arnoldii Leseigneur 125

- neoalienus $\mathrm{n} .121$

- neoarnoldii $\mathrm{n} .125$

Carduus 73

- crispus L. 74

Carex 15, 145

- hirta 132, 134, 145

- hudsonii 83

- pendula

- rostrata 4

- sylvatica 76

Carpomyia incompleta (Becker) 144

Cassinia quinquefaria 240

Catoplatus citrinus Horvath 234

Centaurea 226

- jacea 79,83

- nigra 83

Centorisoma Becker 8, 9

- elegantulum Becker 9, 17

Cerasium avium 144

Cerastium holosteoides 148

Ceratitis capitata (Wiedemann) 144

Cerodonta (Butomyza) caricivora (Groschke) 76

- (Dizygomyza) bimaculata (Meigen) 83

- (D.) caricicola (Hering) = soend 86

- (D.) chaixiana Groschke 83

- (D.) morosa (Meigen) 83, 134

Cerodontha (Butomomyza) scirpi (Karl) 136

- (Butomyza) angulata (Loew) 132, 134, 145

- (B.) caricivora (Groschke) 145

- (B.) eucaricis Nowakowski 132, 145

- (Cerodonta) denticornis (Panzer) 131, 135

- (Dizygomyza) caricicola (Hering) 132

- (D.) caricicola (Hering) $=$ soen $76,83,143$

- (D.) iraeos (Robineau-Desvoidy) 76, 145

- (D.) luctuosa (Meigen) 76

- (Icteromyza) geniculata Fallén 68
- (Phytagromyza) flavocingulata (Strobl) $76,86,145$

- (Poemyza) 82

- (P.) ?incisa (Meigen) 82

- $(P$.) deschampsiae (Spencer) 76, 136

- (P.) incisa (Meigen) 82, 86, 136, 145

- (P.) lapplandica (Rydén) $=$ tatrica 82

- (P.) luzulae Groschke 86

- (P.) pygmaea (Meigen) 76, 82, 136, 145

- denticornis Panzer 70

- luzulae (Groschke) 76

- pygmaea Meigen 138

- silvatica (Groschke) 76,83

Cetema Hendel 8,9

— cereris (Fallén) 11, 12, 15, 19

- elongatum (Meigen) 15

- myopinum (Loew) 15

- neglectum 14

- neglectum Tonnoir 15

- simile Ismay 15

Ceutorhynchus arquatus (Herbst) 5

- melanostictus (Marsham) 5

Charagochilus gyllenhali (Fallén) 221

Chelaria Haworth 35, 36

- triorthias Meyrick 35, 39, 42

Chenopodium 73, 223

- bonus-henricus 151

- polyspermum 148

Chilotrichia Foerster 147

- blandus 147

Chirosia histricina Rondani 151

Chlamydatus (Attus) eurotiae Kerzhner 224

Chlorops Meigen 7, 9, 11

- brevimanus Loew 19

- centromaculatus Duda 18

- distinguendus Frey 18

- fasciatus Meigen 18

- gracilis Meigen 19

- pannonicus Strobl, 18

- planifrons Loew 19

- riparius Smirnov 18

- rossicus Smirnov 19

- rufescens Oldenberg 19

- rufinus Zetterstedt 19

- scalaris Meigen 19

— scutellaris Zetterstedt 19

- serenus 18

- speciosus Meigen 19 
- troglodytes Zetterstedt 19

- varsoviensis Becker 19

- zonulatus Wahlgren 19

Chrysanthemum 234

- cinerarifolium 83

Chylizosoma beckeri Séguy 151

Cirsium 73

- acaulon 82

- eriophorum 136

Clinopodium vulgare 145

Cloeon 51, 52, 56-58

- marginalis 52

Closterotomus costae Reuter 220

Coleophora 167, 168

— adjunctella Hodgkinson 167

- alcyonipennella (Kollar) 167

- alticolella Zeller 167, 168

- discordella Zeller 167

- gallipennella (Hubner) (from Latvi 167

- glaucicolella Wood 167, 168

- lusciniaepennella (Treitschke) 167, 168

- proterella sp.n. 168

- saxicolella (Duponchel) 168

- taeniipennella Herrich-Schäffer 168

- virgaureae Stainton 168

Compsidolon (Apsinthophylus) bipunctatum eremita 225

- (A.) pumilum (Jakovlev) 225

- (Compsidolon) nebulosum (Reuter 225

- (Compsidolon) elegantulum Reuter 225

Conostethus cf. hungaricus Wagner 227

Convolvulus erinaceus 225

Copium teucrii iranum Wagner 234

Cornus 94

Craspedostethus Schwarz 117

— iranicus n. sp. 120, 121

- linnavuorii n. sp. 120, 122

- schusteri (Schwarz) 120, 122

Crataegus 42

Creontiades pallidus (Rambur) 220

Crepis praemorsa 134

Crioceris nigropunctata Lacordaire 209

Crossocerus 247

- congener (Dahlbom) 247

- megacephalus (Rossi) 247

- annulatus 247

— assimilis (F. Smith) 247, 248

- cetratus (Shuckard) 247

Crunoecia irrorata (Curtis) 2
Cryptonastes Foerster 79

- tersus Foerster 79, 80

Cryptonevra Lioy 8, 9

— diadema (Meigen) 9, 13, 14

- flavitarsis (Meigen) 9, 14

Cryptophagus subfumatus $\mathrm{Kr} .50$

Cryptus albulatorius Gravenhorst 176

Cucullia 106

— amota Alphéraky 106

— asteris ([Denis \& Schiffermüller]) 106

- asteroides Guenée 106

- cemenelensis Boursin 106

- convexipennis Grote \& Robinson 106

- dracunculi (Hübner) 103, 106

- elongata (Butler) 106

- florea Guenée 106

- mixta (Freyer) 103

- mixta lorica 103, 104

- mixta mixta 104

- omissa Dod, 1916106

- postera Guenće 106

- virgaureae Boisduval 106

- xeranthemi Boisduval 103, 106

Cuphocera ruficornis Macquart 1

Cupressus 220

Cynachum vincetoxicum 144

Cynomya mortuorum (Linnaeus) 4

Cyperus 219

Cypha imitator (Luze) 50

Cyrtorhinus caricis (Fallén) 223

Cytisus scoparius 145

D

Dactylis glomerata 136, 138, 145

Dacus oleae (Gmelin) 144

Daphne mezereum 74

Dapsilarhtra sulcifera Papp 69

Dasypoda hirtipes (Fabricius) 4

Datura 147

- stramonium 73

Daucus 79

Delia antiqua (Meigen) 150

- brassicae (Hoffmannsegg in Wiedemann) 150

- echinata Séguy 134, 148

- quadripila (Stein) 130, 149

Dendrostellera lesserti 223

Denisia Hübner 250

- similella (Hübner) 249 
- stipella (Linnaeus) 249

Depressaria 35

Deraeocoris (Camptobrochis) punctulatus

(Fallén) 219

- (C.) serenus Douglas \& Scott) 219

- (Knightocapsus) lutescens (Schilling) 219

- (Phaeocapsus) pilipes Reuter 219

Dermestes 245

Deschampsia caespitosa $76,82,86,131,136$, 145

- flexuosa 94

Desmatophorus Thomson 131

Desmiostoma Foerster 152

- parvulum (Wesmael) 67, 152

Diachasma Foerster 152

- rugiventre (Thomson) 152

Dianthus barbatus 136, 148

- caryophyllus 136

Dichrooscytus persicus Josifov 221

Dicronychus Brullé 117

- extinctus (Erichson) 125

- fusivittatus n. sp. 122, 123, 124

- mesopotamicus n. sp. 123, 125

Dictyonota opaca (Linnavuori) 233

Dicyphus (Mesodicyphus) testaceus Reuter 219

- testaceus Reuter 216

Dilophus borealis Skartveit, 1993115

Diplotoxa Loew 8, 18

- messoria (Fallén) 8, 10, 11, 13, 17

Diplotoxoides Andersson 8

- dalmatinus (Strobl) 8, 10, 17

Dizygomyza 82, 136

- (Butomyza) caricivora (Groschke) 132

Dorytomus ictor (Herbst) 3

Dryops anglicanus Edw. 50

E

Elaeagnus 204

- commutata Bernh. ex Rydb 203

Eleagnus angustifolia 222, 227

Eleocharis 17

Elymus (=Agropyron) repens 146

- repens 72, 82, 94

Empria 95

- granatensis Lacourt 96

- hungarica (Konow) 95,96, 97, 99, 100, 101

— impatientis Fischer 67, 68

— pumila (Konow) 95, 96, 97, 98, 99, 100, 101
- pumiloides Lindqvist 95, 96, 97, 98, 99 (= Empria tricornis Lindqvist) 99

- testaceipes (Konow) 96

- tricornis Lindqvist 95, 97, 99

- zacharovi Dovnar-Zapolskij 99

Enallagma cyathigerum

Endasys annulatus (Habermehl) 175, 176

Endelomyia aethiops (Fabricius) 183, 185

Enoplognatha thoracica (Hahn) 6

Entephria caesiata (Denis \& Schiff.) 1

Ephedra 222, 224

- procera 224

Ephemera lineata 171

Ephemerella 58

Ephialtes (Scambus) eucosmidarum Perkins 175

Epichlorops Zetterstedt 7,9

- puncticollis Zetterstedt 9, 19

Epilobium 235

- hirsutum 219

Epirrita autumnata (Borkh.) 2

- dilutata (Denis \& Schiff.) 2

Equisetum arvense 146

- fluviatile 4

Eremurus stenophyllus 222

Eriophorum 17

- latifolium 68

- vaginatum (Nartshuk) 17

Eriophyes 42

Eristalinus aeneus (Scopoli) 1

Eupatorium cannabinum 145

Euphorbia 222

- hirta in Reunion 240

- nicaeensis All 142

Euphranta connexa (Fabricius) 129, 144

Euphrasia 132

Europiella decolor (Uhler, 1893) 224

Eurotia ceratoides 225

Eurystylus bellevoyei (Reuter, 1879) 221

Eurytenes Foerster 144

- abnormis (Wesmael) 67, 144

- campanariae (Fischer) 67, 68, 145

- cubitalis (Fischer) 67, 145

- impatientis (Fischer) 145

- leptostigma Wesmael 146

- macrocerus (Thomson) 146

- vockerothi (Fischer) 67, 146

Eutrichopsis Foerster 85

- munda Foerster 85 
Eutropha Loew 8, 9

- fulvifrons (Haliday) 9, 16, 18

- variegata (Loew) 9, 16

\section{F}

Fallopia convolvulus 73

Festuca gigantea (L.) Vill. 82, 86

- pratensis 145

- sylvatica 82

Filipendula ulmaria $83,87,88$

— vulgaris 131, 134

Fontinalis antipyretica 172

Fraxinus 203, 204, 222, 224

- excelsior L. 82, 203

- rotundifolia 222

G

Galeatus scrophicus Saunders 233

Galeopsis tetrahit 138, 145

Galium sp. 221, 222

- album $=$ mollugo 76,86

- mollugo 83

- sylvaticum 87

Gampsocoris enslini Seidenstücker 235

Gastrosema Fischer 131

Gelechia Hübner 35

- (Chelaria) gibbosella Zeller 39

- gibbosella Zeller 35, 42

- hippophaella 201

Genista tinctoria 79

Gentiana germanica 134

Glaucopterum kareli Wagner 227

- deserticola (Wagner) 227

Glaucopterum pteropyri Linnavuori 227

Glyceria fluitans 69

- maxima 76

Glyptonotus antarcticus 58

Gonepteryx rhamni 127

Gonserrelus 58

Graphoderus cinereus (Linnaeus) 4

\section{H}

Hadractodes Jussila 176

Haedrotoma Foerster 65

Hallodapus (Plagiorhamma) concolor Reuter 218,219

-(P.) pseudoconcolor Linnavuori 218, 219,
223

- costae (Reuter) 223

- suturalis (Herrich-Schaeffer) 223

Halostachys belangeriana 228

Haloxylon 220, 228, 229

- aphyllum 228, 229

- persicum 228, 229

Helianthemum 163

Helianthus 234

Heliotropium 228

Helophorus minutus Fabricius 3

Hemiteles pictipes Gravenhorst 175

Hepatica nobilis 145

Heracleum 138

— sphondylium 72, 79, 82, 86

Hieracium pilosella 133

- sp. sabaudum group 83,86

- umbellatum 82

Hippophaë 204

—rhamnoides L 201, 203, 206

— rhamnoides ssp. rhamnoides L. 201

Holconotus Foerster 69

- comatus 69

Holcus lanatus 69, 76, 82, 131, 135, 138, 145

- mollis 130

Homogyne alpina 82

Honckenya peploides 130, 149

Hordeurn sp. 69, 226, 136

- vulgare 152

Horistus turcomanus (Horváth) 222

Humulus lupulus 88

Hyalopterus pruni (Geoffr.) 237

Hybiscus sabdarifa 240

Hydrellia argyrogenis Becker 68

- cochleariae Haliday 68, 130

- fascitibia Roser 68

- griseola Fallén 84, 130

- potamogeti Hering 68

- stratiotae Hering 68

Hydrochus megaphallus van Berge

Henegouwen 3

Hydroporus puberulus LeConte 3

- semenowi Jakovlev 3

Hydroptila dampfi Ulmer 2

- forcipata Eaton 2

Hydrovatus 179, 181

- wewalkai n. sp. 179, 180

— enigmaticus 179

- opacus Sharp 179 
Hylastes cunicularius Er. 30

Hyoidea notaticeps Reuter, 1876222

Hyoscyamus niger 134, 137, 149

Hypatima Hübner 35

Hypericum perforatum var. angustifolium 240

Hypochoeris aetnensis 134

Hypocynodus Foerster 139

- crassipes Foerster 139

Hypolabis pallipes 134

Phytomyza sphondyliivora Spencer 86

I

Ichneumon difformis Gmelin 245

Idechtis caudatus Gregor 245

Idotarmonides Agajew 117

— bicolor n. sp. $118,123,124$

Idris 187

Impatiens noli-tangere 145

Incisolema cylindricollis (Lacordaire) 213

Ips DeGeer 32, 33

- duplicatus Sahlb. 30

- amitinus Eichh. 29, 30-33

- typographus L. 29, 30, 30-31, 31, 33

Iresine lindeni 71

Iris pseudacorus 76, 145

Viburnum tinus 240

\section{$\mathbf{J}$}

Jacksonella falconeri (Jackson) 1, 6

Jasminum 203

Juglans mandschurica 42,47

Juncus 219, 223, 234

- effusus 76

Juniperus excelsa 221, 222, 234

$\mathbf{K}$

Kainopaeopius Fischer 78

Kalidium 228

Kawabei 48

Knautia arvensis 81,83

Kochia? 228

\section{$\mathbf{L}$}

Lacon unicolor (Candèze) 117, 123, 124

Laemocoris reuteri (Jakovlev) 223

Lamiastrum galeobdolon 145

Lamium album 234
Larix sibirica 250

Lasiargus hirsutus (Menge) 6

Lasiosina Becker 18, 18, 19

- albipila (Loew) 8, 13, 17

- brevisurstylata Dely-Draskovits 8, 13, 17

- cinctipes 17, 18

- herpini (Guerin-Meneville) 10, 13, 17, 18

- parvipennis Duda 7,13, 18

Lasius flavus 94

- niger 94

Lathroplex Förster 241, 245

- anthreni sp. n. 241, 244, 245

- caudatus (Gregor) 245

- clypearis Thomson 241, 244, 245

Lathyrus latifolius 88

- torreyi 132

Lema affinis Clark 213

- aperta Lacordaire 213

- cephalotes Lacordaire 212

- democratica Clavareau 209, 212

- dregei Lacordaire 210

- erythraeana Clavareau 209

- foraminosa Lacordaire 212

- fuscitarsis Jacoby 210

- hottentota Lacordaire 212

- humeralis (Guérin-Ménéville) 212

- kerremansi Clavareau 211

— korschefskyi Heinze 210

- milleriana Wollaston 209, 212, 213

- mulangensis Heinze 210

- nigriventris Gerstaecker 213

- omukama sp.n. 209, 210

- pauli Weise 209, 210

- pauperata Lacordaire 212, 213

- quadrifasciata Heinze 209, 211- 213

- quadripartita Lacordaire 213

- reducteapicalis Pic 211

- restricta Clavareau 209, 210

- rubricollis Klug 211, 213

Lepidargyrus instabilis (Reuter) 225

Leptacinus formicetorum Märk. 50

- pusillus (Steph.) 50

Lepthyphantes decolor (Westring) 6

Leptopterna inopinata Vinokurov 219

Lesteva punctata Erichson 2

Leucanthemum maximum 134

Leucopiargenticollis Zetterstedt 237

Leucopis 237, 239

- argentata Heeger 237 
- compacta Tanas. 239

- formosana 239

- formosana Henning 237, 238

- griseola Fall. 240

- steinbergi Tanas 239

- glyphinivora Tanas. 237

- (Leucopomyia) lubrica Frey 239

Leucopomyia 239

Leucopterum candidatum Reuter 227

Leymus 6

Ligustrum 203

Lilioceris aestivalis (Clark) 211

- africana (Jacoby) 211

- latipennis (Clark) 211

- livida (Dalman) 211

Limnephilus extricatus 2

- hirsutus (Pictet) 2

Liocoris tripustulatus (Fabricius) 221

Liophloeus lentus herbsti Gyllenhal 5

- tessulatus (Müller) 5

Lipara 14

Liriomyza 79, 134, 142

- amarellae Hering 134

- amoena (Meigen) 83, 138

- approximata (Hendel) 74

- balcanica (Strobl) 142, 145

- bruscae Hering 146

- bryoniae (Kaltenbach) 134

- centaureae Hering 81, 83, 134

- congesta (Becker) 74, 79, 133, 137, 141

- demeijerei Hering 145

- eupatoriana Spencer 134, 145

- eupatorii Kaltenbach 138

- fasciola 152

- flaveola (Fallén) 81, 82, 141, 145

- morio (Brischke) 87

- pascuorum (Meigen) 83

- pisivora Hering 80

- pusilla Meigen 82, 133

_pusio (Meigen) 76, 132 = graminicola de Meijere 86

- scorzonerae Rydén 145

- strigata (Meigen) 79, 134, 141

Liriomyza strigata Meigen 72

- taraxaci Hering 134

- wachtli Hendel $=$ veratri Groschke 134

Lissosema Fischer 131

Listera ovata 85
Lita lepigreella Lucas 35, 39

Lonchaea nigra Meigen 73

Lonicera 144, 203

- caerulea L. 203

- caprifolium L. 80, 203

- orientalis 83

- periclymenium L. 82, 83, 138

- sp. (orientalis

= ambigous!) 74

- tatarica L. 205

- xylosteum 82

Lotus 163

Lucilia caesar (Linnaeus) 71

Luzula pilosa 83

- sylvatica 76, 86

Lychnis coronaria 136

Lycium depressum 225, 234

Lygus gemellatus gemellatus (Herrich-

Schaeffer) 221

— pratensis pratensis (Linnaeus) 221

- rugulipennis Poppius 221

M

Macrolophus epilobii Puchkov 219

- melanotoma (A. Costa) 219

- pygmaeus (Rambur) 219

Macrosyphum rosae (L.) 240

Macroteleia sp. 187

Macrotylus (Alloeonycha) paykullii (Fallén) 223

Malacocoris chlorizans (Panzer) 222

Malus 42

Mastrus pictipes (Gravenhorst) 175

— tenuicosta (Thomson) 175

Maurodactylus albidus (Kolenati) 228

- nigrigenis (Reuter) 228

Medicago sativa 79, 133

- sativum 135

Megacoelum brevirostre Reuter 220

Megaloceroea recticornis (Geoffroy) 220

Megalocoleus molliculus (Fallén) 226

Melandrium album 136

- rubrum 136

Melanotus Eschscholtz 117

— dichroides n. sp. 119, 120, 123, 124

- dichrous (Erichson) 119

- fulvus Reitter 119

- iranicus n. sp. 119, 120, 123, 124

— turkmenicus Dolin \& Ata-muradov 121 
Melanum Becker 7,9

- laterale Haliday 9, 10, 19

Melica nutans 136

Mentha 219

Meromyza Meigen 7, 8, 11, 19

- rostrata Hubicka 18

- saltatrix L. 19

- elbergi Fedoseeva 18

Metacanthus meridionalis (Costa) 234

Metopobactrus prominulus (O.P.-Cambridge) 6

Metopomyza violiphaga (Hendel) 83

Micaria nivosa L. Koch 6

Microcryptus puncticollis Thomson 176

Mirificarma Gozmány 135

Misophthora Foerster 80

- laevigata Foerster 80

Monocris griseolus Puchkov 227

Monosteira discoidalis (Jakovlev) 234

- inermis Horváth 234

- unicostata (Mulsant \& Rey) 234

Musca domestica Linnaeus 71

Muscina stabulans Fabricius 149

Myiolia caesio Harris 134

Myosotis laxa 143

- palustris 143

- sylvatica 82

Myriophyllum spp. 172

Myrmecophyes alacer Horváth 222

— variabilis Drapolyuk 222

\section{$\mathbf{N}$}

Napomyza evanescens (Hendel) 87, 133, 137,140

- nigritula (Zetterstedt) = cineracea (Hend) 133, 139, 140

- nigritula (Zetterstedt) = Phytomyza ciner 137

- salviae Hering 132

Nasocoris albipennis Lindberg 224

- argyrotrichus Reuter 224

Neides afghanus Seidenstïcker 234

- brevipennis Puton 234

Nemeritis macrocentra (Gravenhorst) 245

Nemorimyza posticata (Meigen) 85, 86

Neohaplegis Beschovski 9

- tarsata 9, 13

Neopius carinaticeps Gahan 69

Nosopaeopius Fischer 78

Nosopaeus (Opius) polyzonius 72
Nosopoea Foerster 85

- cingulatus 86

- stramineipes 86

Notostira poppiusi Reuter 220

O

Odontites verna 142

Odontopoea Fischer 129

Oedemera croceicollis (Gyllenhal) 4

Olea europea 144

Oncophanes flaviceps 65

- flaviceps Hellén 70

Oncotylus (Cylindromelus) setulosus (HerrichSc 225

- (Oncotylus) affinis Jakovlev 226, 227

- (O.) vitticeps Reuter 226

Ophiomyia 82, 133, 135

- maura (Meigen) 76, 83, 87, 132

- pulicaria (Meigen) 81

Opiognathus Fischer 73

Opiothorax Fischer 139

Opius Wesmael 65, 66, 68, 131, 144

- (Cryptonastes) minor Fischer 67

- (A) saevus Haliday 67

- (Adontopius) adentatus Fischer 66, 67, 139

-(Agnopius) austriacus Fischer 67, 81

- (A.) basirufus Fischer 81

- (A.) heringi Fischer 67, 82

- (A.) rex Fischer 82

-(A.) similis Szépligeti 67,83

- (A.) similoides Fischer 67,83

-(A.) tirolensis Fischer 67, 84

- (Allophlebus) fuscipennis Wesmael 67, 137

- (A.) padidalis Fischer 67, 137

- (A.) pulcherrimus Fischer 67, 137

- (A.) pulchriceps Szépligeti 138

- (A.) singularis (sensu Fischer, nec Wesmael) 138

- (A.) singularis Wesmael 138

- (A.) sitagrus Papp 138

- (A.) staryi Fischer 67, 68, 138

- (A.) tarni Papp 138

-(A.) victus Haliday 138

- (Allotypus) altimontanus 85

-(A.) ambiguus 86

-(A.) austriacus 81

- (A.) basirufus 81

-(A.) caprifoliae 80

-(A.) celsus 86 
- (A.) cingulatus 86

- (A.) circulator 87

- (A.) connivens 129

- (A.) cubitalis 145

- (A.) diabolicus 89

- (A.) geniculatus Thomson 84

- (A.) heringi 82

- (A.) instabilis 80

- (A.) irregularis Wesmael 84

- (A.) iuxtangelum 81

- (A.) leptostigma 146

- (Allotypus) macrocerus Thomson 146

- (A.) maculipes 87

- (A.) mundus 85

- (A.) pirchitticola 88

- (A.) pulicariae 81

- (A.) punctiventris Thomson 130

- (A.) rex 82

-(A.) saevulus Fischer 67, 85

- (A.) saevus Haliday 85

- (A.) seebensteinensis 88

- (A.) sigmodus 88

- (A.) similis 83

- (A.) similoides 83

- (A.) speciosus 88

- (A.) tirolensis 84

- (A.) tscheki 78

- (A.) tuberculifer 88

- (A.) viennensis 89

- (Apodesmia) aethiops Haliday 67, 70

- (A.) fulvicollis Thomson 66, 70

-(A.) karesuandensis Fischer 67, 71

- (A.) nitidulator (Nees) 66, 68, 71

- (A.) ocellatus Wesmael 71

- (A.) polyzonius Wesmael 67,72

- (A.) reconditor Wesmael 72

- (A.) ruficeps Wesmael 66, 72

- (A.) rufipes Wesmael 66,73

- (A.) zelotes 77

- (Aulonotus) comatus Wesmael 69

- (A.) holconotus Fischer 67, 69

-(A.) pilosiscutum Fischer 65, 66, 67, 70

- (Biosteres) blandus 147

- (B.) brevipalpis 147

-(B.) brevisulcus 150

-(B.) carbonarius 150

- (B.) caudatulus 151

-(B.) clypealis 147

- B.) colorativentris Fischer 151
-(B.) haemorrhoeus 148

- (B.) magnicornis 151

- (B.) placidus 151

-(B.) punctiscuta 148

-(B.) rusticus 148

-(B.) scabriculus 149

- (B.) spinaciae 152

- (B.) ultor 149

- (Chilotrichia) blandus 147

-(C.) brevipalpis 147

-(C.) clypealis 147

-(C.) haemorrhoeus 148

-(C.) hyoscyamiellus Viereck 152

-(C.) punctiscuta 148

- (C.) rusticus 148

-(C.) scabriculus 149

- (C.) ultor 149

-(C.) wesmaelii 149

- (Cryptonastes) aureliae 78

-(C.) campnariae 145

-(C.) crassicrus 78

- (C.) curvatus Fischer 79

-(C.) flavigaster 78

-(C.) gracilis Fischer 67, 79

- (C.) gracilis Fischer var. minor 79

- (C.) impatientis 145

- (C.) minor Fischer 79

- (C.) pygmaeus Fischer 67, 79

-(C.) radialis Fischer 80

- (C.) tersus (Foerster) 65, 67, 80

-(C.) vockerothi 146

- (Desmatophorus) abscissus Thomson 133

-(D.) breviscapus Thomson 132

- (D.) piceus Thomson 132

-(D.) pygmaeator 135

- (Diachasma) 152

-(D.) placidus 151

- (D.) rugiventris 152

- (Eutrichopsis) mundus (Foerster) 85

- (Gastrosema) docilis Haliday 131

-(G.) rudiformis 130

- (Hypocynodus) crassipes Wesmael 67, 139

- (H.) latipes Fischer 67, 139

- (H.) mongolaltaiensis Fischer 67, 139

- (Jucundopius) campanariae 145

- (J.) impatientis 145

- (J.) vockerothi Fischer 146

- (Kainopaeopius) crassicrus Thomson 67, 78

- (K.) speciosus Fischer 67 
-(K.) tscheki Fischer 67, 78

- (Lissosema) parvungula Thomson 67, 131

- (Melanotrichus) problematicus Linnavuori 215

- (Misophthora) austriacus 81

-(M.) basirufus 81

- (M.) caprifolii Fischer 66, 67

- (M.) caprifolii Fischer 80

- (M.) fallax 81

- (M.) heringi 82

-(M.) instabilis Wesmael 80

-(M.) iuxtangelum Fischer 66, 67, 81

- (M.) pulicariae Fischer 81

- (M.) rex 82

- (M.) similis 83

-(M.) similoides 83

- (M.) tirolensis 84

- (Nosopaeopius) aureliae Fischer 67

- (N.) aureliae Fischer 78

- (N.) crassicrus 78

- (N.) flavigaster Fischer 67, 78

- (Nosopaeus) connivens Thomson 129

- $(N$.) dentifer Thomson 86

- (N.) stramineipes Thomson 86

-(N.) altimontanus Fischer 67, 85

-(N.) ambiguus Fischer 86

- (N.) ambiguus Wesmael 86

- (N.) celsus Haliday 67, 86

- (N.) cingulatus Wesmael 86

- (N.) circulator (Nees) 67, 68, 87

- (N.) cubitalis 145

- (N.) diabolicus Fischer 67, 89

- (N.) eversus Papp 87

- (N.) macrocerus 146

- (N.) maculipes Wesmael 67, 68, 87

-(N.) mundus 85

- (N.) pirchitticola Fischer 66, 67, 88

- (N.) seebensteinensis Fischer 88

- (N.) sigmodus Papp 66, 67, 88

- (N.) speciosus Fischer 88

- (N.) tuberculifer Fischer ssp. tubercul 88

- (N.) seebensteinensis Fischer 67

- (N.) tuberculifer Fischer 67, 88

- (N.) tuberculifer Fischer ssp. diabolicus Fisch 67, 88, 89

- (N.) viennensis Fischer 67, 89

- (Nosopoeaopius) tscheki Fischer 78

- (Nosopoeus) polyzonius 72

$-(N$.) rudis 69
- (Odontopoea) connivens Thomson 129

-(O.) connivens Thomson 67

- (O.) epulatus Papp 129

- (O.) epulatus Papp 66

- (O.) epulatus Papp 67

-(O.) paranivens Fischer 67, 130

- (Opiognathus) pactus Haliday 67, 73

- (O.) propodealis Fischer 73

- (Opiostomus) leptostigma 146

- (Opiothorax) abditiformis Fischer 67, 139

- (O.) adentatus Fischer 139

- (O.) affectus Papp 136

-(O.) attributus Fischer 139

- (O.) caudifer 141

-(O.) dureseaui Fischer 67, 140

- (O.) filicornis Thomson 68, 140

- (O.) funebris Wesmael 67, 140

- (O.) inflammatus Fischer 67, 140

- (O.) levis Wesmael 68, 141

- (O.) longicornis Thomson 67, 68, 141

- (O.) magnicauda Fischer 67, 68, 142

- (O.) minusculae Fischer 67, 142

- (O.) mirabilis Fischer 67, 142

- (O.) nigricoloratus Fischer 143

- (O.) opacus Fischer 67, 143

- (O.) soenderupianus Fischer 67, 143

- (O.) spretus Haliday 68, 143

- (O.) turcicus Fischer 67, 143

- (O.) agromyzicola Fischer 67, 68, 131

- (O.) breviscapus Thomson 67, 132

- (O.) caricivorae Fischer 67, 132

- (O.) circinus Papp 66, 67, 132

- (O.) comatus Wesmael 69

- (O.) exilis Haliday 132

- (O.) fasciatus Thomson 75

- (O.) gigapiceus Fischer 133

- (O.) gigapiceus Fischer 67

- (O.) lugens Haliday 133

- (O.) meracus Fischer 133

-(O.) pallipes Wesmael 68, 131, 134

- (O.) peterseni Fischer 134

- (O.) piceus 132

- (O.) pygmaeator (Nees) 135

- (O.) subpallipes Tobias 67, 135

- (Pendopius) cisromensis Papp 132

- (P.) exilis 132

- (P.) longicornis 141

- (P.) pendulus Haliday 67, 68, 137

- (Phaedrotoma) crassipes 139 
- (P.) adentatus 139

- (P.) caudifer 142

- (P.) depeculator (Foerster) 135

- (P.) diversus Szépligeti 67, 68, 135

- (P.) dureseaui 140

- (P.) exiguus Wesmael 136

- (P.) exilis 132

-(P.) filicornis 140

- (P.) funebris 140

- (P.) fuscipennis 137

- (P.) inflammatus 140

- (P.) latipes 139

- (P.) levis 141

- (P.) longicornis 141

- (P.) magnicauda 142

- (P.) minusculae 142

- (P.) mirabilis 142

-(P.) nigricoloratus 143

- (P.) opacus 143

- (P.) pendulus 137

- (P.) pulcherrimus 137

-(P.) pulchriceps 138

- (P.) pulchriventris Fischer 67, 136

- (P.) soenderupianus 143

- (P.) spretus 143

- (P.) staryi 138

-(P.) turcicus 143

- (P.) variegatus Szépligeti 136

-(P.) attributus 140

- (Pseudopius) extusus Papp 132

- (Psyttalia) concolor 144

- (P.) rhagoleticola 144

- (P.) rhagoleticolus 144

- (Rhabdospilus) areolaris Thomson 72

- (Stigmatopoea) cubitalis 145

- (S.) macrocerus 146

- (Stomosema) gyoerfii Fischer 130

-(S.) gyoerfii Fischer 66

- (S.) mutus Fischer 130

- (Therobolus) caudatus 74

$-(T$.$) docilis 131$

- (T.) exsertus Thomson 74

-(T.) pactus 73

- (T.) parvungula Thomson 131

- (T.) rotundiventris Thomson 76

- (T.) rufipes 73

- (T.) trisulcus Thomson 77

- (T.) truncatus 77

- (Tolbia) caesus Haliday 130
$-(T$.$) docilis 131$

-(T.) parvungulus 131

-(T.) rudiformis Fischer 68, 130

- (Utetes) aemulus Haliday 67, 74

- (U.) brutus Papp 72

-(U.) caudatus Wesmael 67,74

- (U.) coracinus Thomson 67, 75

-(U.) curtipectus Fischer 66, 67, 75

$-(U$.) exsertus 74

- (U.) fasciatus Thomson 75

-(U.) hilaris Fischer 75

-(U.) hostium 75

- (U.) insertus 77

-(U.) magnus Fischer 67, 76

-(U.) ochropus Thomson 67, 76

-(U.) rotundiventris Thomson $65,67,76$

- (U.) trisulcus Thomson 67,77

- (U.) truncatus Wesmael 77

- (U.) zelotes Marshall 67,77

-(Xynobius) aemulus 74

-(X.) aethiops 70

- (X.) caudatus 74

- (X.) comatus 69,70

-(X.) coracinus 75

-(X.) curtipectus 75

-(X.) decoratus Stelfox 67,68

- (X.) fasciatus 75

- (X.) flaviceps 69

-(X.) fulvicollis 70

- (X.) hilaris 75

-(X.) holconotus 69

-(X.) karesuandensis 71

-(X.) magnus 76

-(X.) nitidulator 71

- (X.) notabilis Fischer 67,69

- (X.) ocellatus 72

- (X.) ochropus 76

- (X.) pactus 73

- (X.) pilosiscutum 70

-(X.) polyzonius 72

$-(X$.$) propodealis 73$

- (X.) reconditor 72

-(X.) rotundiventris 76

-(X.) rudis Wesmael 67,69

$-(X$.$) ruficeps 72,73$

-(X.) sayanicus Tobias 76

-(X.) trisulcus 77

-(X.) truncatus 77

- (X.) zelotes 77 
- $[A]$ reconditor Wesmael 66

- [H.] latipes Fischer 66

- abnormis Wesmael 144

— abscissus 133

- addendus Fischer 87

- adveniens Fischer 133

— aemulus Haliday 74

— aethiops Haliday 70

- albicoxis Marshall 84

- altimontanus Fischer 85

— ambiguus Wesmael 86

— aquaticus Muesebeck 130

- arenosus Szépligeti 143

— attributus Fischer 139

- aureliae Fischer 78

- austriacus Fischer 81

- basirufus Fischer 81

- bipustulatus Fischer 84

— blandus Haliday 147

- borealis 147

— brevipalpis Thomson 147

- brevisulcus Thomson 150

- brusceanus Fischer 146

- caesus Haliday 130

- campanariae Fischer 145

- caprifolii Fischer 80

- carbonarius Wesmael 149, 150

- carinatus 144

- castaneiventris Thomson 148

- caudatulus Thomson 150

- caudatus Wesmael 74

- caudifer Fischer 141

- celsus Haliday 86

- cingulatus Wesmael 85, 86

- circulator 87

- clarus Haliday, 143

- clypealis Thomson 147

- comparandus Fischer 75

- concolor Szépligeti 144

- connivens 129

- consors Fischer 80

- coracinus Thomson 75

- crassicrus Thomson 78

- crassipes Wesmael 139

- cubitalis Fischer 145

— cupidus Gahan 71

- curtipectus Fischer 75

- curvatus Fischer 79

— decoratus Stelfox 68
- depeculator 135

- diabolicus Fischer 88

- dilatatus Fischer 140

- diversipes 135

- diversus 136

- diversus Szépligeti 135

- docilis Fischer 131

- docilis Haliday 131

- epulatus Papp. 129

- exiguus Wesmael 135, 136

- exsertus 74, 77

- fallax Szépligeti 81

- filicornis Thomson 140

- flaviceps Gahan 70

- flavigaster Fischer 78

- foveola Fischer 129

- fulgidus Haliday 152

- fulvicollis Thomson 70

- funebris Wesmael 140

- fuscipennis Wesmael 137

- fuscitarsis Szépligeti, 144

- geniculatus Thomson 84

- gracilis Fischer 79

- gyoerfii 130

- györfii Fischer 130

- haemorrhoeus Haliday 148

- heringi Fischer 82

- hians Stelfox 146

- hilaris Fischer 75

- holconotus Fischer 69

- hostium Fischer 75

- hungaricus Szépligeti 72

- hydrelliae Muesebeck 130

- hydrelliae Rimsky-Korsakow 130

- hydrellianus Fischer 130

- impatientis Fischer 145

- impressus Wesmael 150

- inflammatus Fischer 140

- insertus Fischer 77

— instabilis Wesmael 80

— irregularis Wesmael 84

- jonaitisi Jakimavicius 149

- karesuandensis Fischer 71

- latipes Fischer 139

- leptostigma Wesmael 146

- leptostigmus 146

- levis Wesmael 139, 141

- longicornis Thomson 141

- longipes Fischer 86 
- macrocerus 146

- maculipes Wesmael 87

- magnicauda Fischer 142

- magnicornis Wesmael 151

- magnus Fischer 76

- melanocerus Wesmael 152

- melanosoma Szépliegti 150

- melbus Papp 74

- minor Fischer 79

- minusculae Fischer 142

- mirabilis Fischer 142

- moldavicus Jakimavicius 148

- monticola Szépligeti 81

- mundus 85

- mutus Fischer 130

- nigricinctus Fischer 78

- nigricoloratus Fischer 143

- nitidulator 66, 71

- nodatus Fischer 81

- notabilis Fischer 69

- nudiscutum Fischer 152

- obscurus Szépligeti 133

- ocellatus Wesmael 71

- ochrogaster Wesmael 78

- ochropus Thomson 76

- opacus Fischer 143

- pactus Haliday 73

- pallidipes Marshall 134

- parvulus Wesmael 152

- parvungula Thomson 131

- pegomyiae Gahan 152

- pendulus Haliday 137

- perproximus Silvestri 144

- phytomyzae Fischer 86

— pilosiscutum Fischer 70

- placidus Haliday 150, 151

- polyzonius Wesmael 72

- procerus Wesmael 150

- propodealis Fischer 73

- pulcherrimus Fischer 137

- pulchriceps Szépligeti 138

- pulchriventris Fischer 136

- pulicariae 81

- pulicariae Fischer 81

- pumilio Wesmael 131

- punctiscuta Thomson 148

- punctiscutis 148

- pygmaeus Fischer 79

- radialis Fischer 80
- reconditor Haliday 75

- reconditor var. 3, Wesmael 76

- reconditor Wesmael 72

- reptantis Fischer 86

- rhacoleticola 144

— rhagoleticolus Sachtleben 144

- rotundiformis 130

- rotundiventris 76

- rudiformis Fischer 130

— rudis Wesmael 69

- ruficeps Wesmael 70,72

- rufipes Wesmael 66, 72, 73

- rugiventris Thomson 152

- ruminans Fischer 140

- rusticus Haliday 148

- saevulus Fischer 85

- saevus Haliday 85

- scabriculus Wesmael 148

- scrutator Tobias 76

- seebensteinensis Fischer 88

- semiaciculatus Stelfox 135

- sigmodus Papp 88

- silvicola Szépligeti 75

- similiformis Fischer 83

- similis Szépligeti 81, 83

- similoides Fischer 83

- singularis (sensu Fischer, nec Wesmael) 138

- soenderupianus Fischer 143

- speciosus Fischer 88

- spinaciae Thomson 152

- spretus Haliday 143

- staryi Fischer 138

- subtilis Szépligeti 130

- tarsator Thomson 152

- tersus 80

- testaceus Wesmael 74

- tirolensis Fischer 84

— trisulcatus 77

- truncatus Wesmael 77

- tuberculifer Fischer 88

- turcicus Fischer 143

- turcmenicus Fischer 87

- variegatus Szépligeti 136

- varipes Szépligeti 141

- wesmaeli 149

- wesmaelii Haliday 149

— victus Haliday 138

- victus = singularis sensu Fischer 137 
— viennensis Fischer 89

- vittatus Ruschka 71

Orchis mascula L. 85

Oresbius puncticollis (Thomson) 175, 176

Orthocephalus bivittatus Fieber 222

- modarresi Linnavuori 222

Orthops (Montanorthops) pilosulus (Jakovlev) 221

- (Orthops) frenatus (Horváth) 221

- (O.) kalmi (Linnaeus) 221

Orthotylus (Melanotrichus) flavosparsus (C.R. Sahlberg) 223

-(M.) eleagni Jakovlev 215, 222

- (M.) fieberi fieberi (Frey-Gessner) 222

-(M.) hirtulus Wagner 223

-(M.) minutus Jakovlev 223

-(M.) nymphias Linnavuori 223

-(M.) pusillus Reuter 223

- (M.) rubidus (Puton) 223

- (M.) rudbaricus Linnavuori 217

- (M.) turcmenorum Puchkov 217, 223

- (M.) viridissimus Linnavuori 223

- (Orthotylus) prunicola Linnavuori 222

- (Parapachylops) putshkovi Josifov 215, 222

- eleagni Jakovlev 215, 217, 218

— problematicus J. Sahlberg 217

- problematicus Linnavuori 215, 218

- putshkovi Josifov 215, 216, 217

- rudbaricus 217, 218

- rudbaricus Linnavuori $=O$. turcmen 215

- turcmenorum 218

Oulema punctatissima (Heinze) 213

Oulema raffrayi (Chapuis) 213

Ozyptila westringi (Thorell) 6

$\mathbf{P}$

"Phora tuberorum Goureau" (="Phora tuberum") 146, 147

Pantolyta Förster 187, 188

— antiqua sp. n. 187,188

- somnulenta Maneval 188

Parallelomma (= Chylizosoma) vittatum (Meigen) 85

Paraphytomyza buhri (de Meijere) 76, 83, 86

- fulvicornis (Hendel) 65

- fulvicornis (Hendel)

$=$ langei $($ Herin 77

- hendeliana
- heringi (Hendel) 82

- luteoscutellata (de Meijere) 72, 74

- luteoscutellata (de Meijere) $=$ xylos 79, 80, 145

- similis (Brischke) 129

- tremulae (Hering) 134, 138

Pardosa palustris (Linnaeus) 6

Parectecephala Becker 9

- longicornis Fallén 9

Parietaria 225

Pastinaca sativa L. 72, 85, 247

Pedicularis palustris 82

Pegomya acetosae Robineau-Desvoidy 73

- betae (Curtis) 66, 71, 73, 149, 150, 152

Pegomya bicolor (Hoffmannsegg in Wiedemann) 134,148

- esuriens-group 71

- holosteae (Hering) 74, 77, 148

- hyoscyami (Panzer) 66, 71, 73, $137,149,150,151,152$

- nigrisquama (Stein) 150

— nigritarsis (Zetterstedt) 71, 73, $134,148,149,150,152$

- setaria (Meigen) 71, 73, 149

- sp. esuriens-group 147, 148

- steini Hendel 150

- terebrans (Rondani) 73

Pendopius Fischer 137

Petasites albus 82

Peucedanum cervaria 79

- oreoselinum 134

- ostruthium 138

- palustre 134

Phaedrotoma Fischer 66

- Foerster 65, 135

- aethiops 74

- depeculator Foerster 135

Phalaris arundinacea 82, 86, 131

Philanthus triangulum (Fabricius) 3

Philophylla heraclei (Linnaeus) 72, 73, 85, 87, 134

Phleum pratense 136, 145

Phlomis 222

Phorbia 148

Phragmites 223

Phragmites australis 14, 72, 82, 130, 131, 237

Phthorophloeus spinulosus Rey 32

Phygadeuon albulatorius (Gravenhorst) 176

— leucostigmus Gravenhorst 176 
- occisor Habermehl 175, 176

- tenuicosta Thomson 175

- triannulatus Gravenhorst 176

Phyllitis scolopendrium 76

Phytagromyza xylostei 72

Phyteuma orbiculare 82

Phytobia 77

Phytocoris (Eckerleinius) crito Linnavuori 220

- (Eckerleinius) eileithyia Linnavuori 220

- (E.) transcaspicus Stichel 220

-(E.) undulatus Reuter 220

- (Exophytocoris) scitulus Reuter 220

Phytoliriomyza hilarella (Zetterstedt) 136

- melampyra (Loew)

= impatientis $($ Bri 145

- variegata (Meigen) 145

Phytomyza 82, 132, 134, 136, 152

- abdominalis Zetterstedt 145

- actaeae Hendel 138

- adjuncta Hering 80

— affinis Fallén 82, 132

- agromyzina Meigen 136

- albimargo Hering 145

- alpina Goureau 86

— alpina Groschke 83

- angelicae Kaltenbach 76, 79, 83, 134

- angelicae Kaltenbach = aegopodii Hendel 86

— angelicastri Hering 79, 86, 138

— anthrisci Hendel 138

- aprilina Goureau $=$ lonicerella (Hendel) 82, 83

- aquilegiae Hardy 138

— astrantiae Hendel 82,83

- atricornis Meigen 79, 81, 83, 133

- bellidina Hering 82, 83, 88

- campanariae Nowakowski 145

- campanulae Hendel 82, 83

- cineracea Hendel 66

- diversicornis Hendel 76

- fallaciosa Brischke 134, 145

- ferulae Hering 79, 134

- flavicornis Fallén 84

- glechomae Kaltenbach 141

- griffithsi Spencer 135

- hendeli Hering 65,80

- heracleana Hering 79, 82, 138

- homogyneae Hendel 76, 82

- hoppi Hering 82
- isais Hering 142

- kyffhusana Hering 134

- lappae Goureau 132

- lappae Goureau = lappina Robineau-Desvoi 83, 86

- leucanthemi Hering 134

- lonicerae Robineau-Desvoidy 82, 83

- lonicerae Robineau-Desvoidy $=$ xylostei 82,83

- marginella Fallén = sonchi Robineau-Desv 83, 86

— milii Kaltenbach 70, 82, 86

- minuscula Goureau 134, 138, 142

- montana Groschke 76, 86

- myosotidis Nowakowski 82

- obscura Hendel 134, 145

- obscurella Fallén 79, 82, 83

— origani Hering 79

- pauliloewi Hendel 134

- periclymenii de Meijere 82, 83, 134

- plantaginis Robineau-Desvoidy 135

- primulae Robineau-Desvoidy 130

- pullula Zetterstedt = matricariae Hendel 134

- pulmonariae Nowakowski 145

- pulsatillae Hering 79

- ramosa Hendel 76, 81, 83, 135

- ranunculi (Schrank) 81, 88, 134

— ranunculi (Schrank) ssp. stolonigena Her 74

- scolopendrii Robineau-Desvoidy 76

- scotina Hendel 80, 140

— sedicola Hering 74, 76, 83

— senecionis Kaltenbach 74, 82, 145

— silai Hering 79

— solidaginis Hendel 83

- sonchi Robineau-Desvoidy 82

— sphondylii Robineau-Desvoidy 79, 134

- succisae Hering 135

- tanaceti Hendel 83, 134

- tenella Hering 82

— thysselini Hering 134

— tussilaginis Hendel 76, 82, 83

- virgaureae Hering 81, 83

Picea abies 250

Picris hieracioides 81,82

- brassicae 127

Pilophorus confusus (Kirschbaum) 223

- betulinus 3

Pistacia vera 224 
Pisum sativum 79, 80, 144

Plagiognathus bipunctatus Reuter 224

Plagiorhamma concolor Reuter 218

- pseudoconcolor Linnavuori 218

Plantago lanceolata 135

- major L. 203

- media 135

Platycephala Fallen 8

- planifrons (Fabricius) 8, 10, 11, 14

- umbraculata (Fabricius) 8, 10, 14

Platydracus chalcocephalus (F.) 50

Platysoma compressum (Herbst) 2

Plectocryptus hilarius Schmiedeknecht 176

Pleuroxonotus nasutus Reuter 226

Poa alpina 82

- trivialis 84

Poecilosoma hungarica Konow 99

Polistes 191, 194, 195, 196

- biglumis (Linnaeus) 191-194, 196

- biglumis (P. bimaculata Geoffroy; P. dubia Kohl; P. kohli Dalla Torre) 193

— dominulus (Christ) 191, 192, 194, 196

— dominulus (P. gallicus auct.) 194

— dubius Saussure 192

- foederatus Kohl 194

— gallicus (Day) 191, 194

— gallicus var. biglumis 192

- nimpha (Christ)

191, 192, 193, 194, 195, 196

— opinabilis Kohl 191

Polygonatum multiflorum 151

Polygonum 73

Polymerus (Poeciloscytus) brevicornis Reuter 221

- (Poeciloscytus) cognatus (Fieber) 221

- (P.) lammesi Rinne 222

- (P.) vulneratus (Panzer) 221

Polyrhembia Foerster 107, 110

Populus 234

- alba 234

- balsamifera 76

— berolinensis 134

- nigra 74

- tremula 87, 138

— tremula L. x tremuloides Michx. 247

Potamanthus 172

- luteus L. 171, 172

Potamogeton 68

- crispus 68
- lucens 68

- perfoliatus 68

Potentilla repens 81

Priobium carpini (Hbst.) 50

Pronototropis punctipennis (Fieber) 226

Prosopis farcta 223, 234

Psallopsis basalis Reuter 228

- bisulcis Linnavuori 228

- caspia Konstantinov 228

— halostachydis Puchkov 228

- haloxyli Puchkov 228

— kaliidicola Konstantinov, 1997228

— kirgisicus (Becker) 228

— similis Wagner 228

Pseudoclavellaria amerinae (Linnaeus) 3

Pseudoloxops iranicus Kerzhner, 1962222

Pseudonupedia abbreviata (Pokorny) 73

Pseudopachychaeta Strobl 8, 18

- approximatonervis (Zetterstedt) 8, 12, 17

- heleocharis (Nartshuk) 8, 11, 12, 17

- ruficeps (Zetterstedt) 8, 17, 19

Pseudoperichaeta nigrolineata (Walker) 1

Psoricoptera Stainton 35, 36, 41, 42, 47, 48

- gibbosella (Zeller) 35-37, 39, 40, 41, 42,4448

- kawabei sp. n. $35,41,45-48$

- latignathosa 35, 38, 42, 44, 45, 47, 48

- lepigreella 42

- speciosella Teich $35,36,40,41,42,44,45$, 47,48

- triorthias (Meyrick) 35, 39, 42

Psyttalia Walker 144

- carinata (Thomson) 129, 144

- concolor (Szépligeti) 67, 129, 144

- rhagoleticola (Sachtleben) 67, 144

Pteridium aquilinum 136, 151

Pteropyrum aucheri 221, 227

Ptinus subpillosus Sturm 50

Pulmonaria angustifolia 145

Pulsatilla pratensis 145

Q

Quasimus minutissimus (Germar) 121

Quedius longicornis $\mathrm{Kr} .50$

Quercus 42, 44, 47, 175, 248

- ilex 42

- mongolica 42

- robur L. 247 


\section{$\mathbf{R}$}

Ramonda ringdahli (Villeneuve) 1

Ranunculus acris 81, 133, 134, 137, 139, 140

- acris L. [fide Fischer] 66

- bulbosus 137

- lanuginosus 87, 133, 137, 140

- repens 74, 88, 145

Reuteria kiritshenkoi Muminov 222

Reuterista unicolor Rosenzweig 221

Rhabdospilus Foerster 150

- placidus 151

Rhagoletis 147

- batava Hering 201

- cerasi (Linnaeus) 76, 144

- meigeni Loew 76

Rhus chinensis Mill. 48

Ribes spicatum E. Robson 203

Rogas decrescens 68

- mutuator 68

Rubiomyza hendeliana Hering 138

- periclymenii Meijere 138

- xylostei (auct.) 74

- xylostei Robineau-Desvoidy [fide Rondani 66

Rumex 148, 152

- acetosa 150

- acetosella 152

S

Salix 42, 44, 45, 47, 77, 219, 223, 224

- alba L. 76

- caprea 250

- cf. caprea 44

Salsola 228

- laricina 228

- richteri 224

Salvia pratensis 80,140

- verticillata 132

Sambucus nigra 83

Saponaria officinalis 136

Scambus eucosmidarum (Perkins) 175

Scaptomyza apicalis Hardy 132, 134

- graminum Fallén 130, 132, 134

Schiffermuelleria obscurella (Brandt 250

- stroemella (Fabricius) 250

Scirpus lacustris 4

- sylvaticus 136

Scleranthus 163

Scorzonera humilis 145
- purpurea 79

Scrophularia nodosa 137

Scythris annae Bengtsson 161, 163, 164

- laminella (Denis \& Schiffermüller) 161

- mariannae Bengtsson 161, 163, 164, 165, 166

- saarelai $161,162,163,164$

- tributella (Zeller) 162

- ventosella Chrétien 163

Secale cereale 88,131

Sedum maximum 83

- telephium ssp. maximum 74,77

Seidlitzia rosmarinus 228

Senecio jacobaea L. 83, 86

- nemorensis 74, 145

- nemorensis fuchsii 82, 134

Sesleria tatrae 82

Sigrisma cylindrica (Klug) 209

Silaum silaus 79

Silene latifolia ssp. alba 80

Silis ruficollis (Fabricius) 3

Solenoxyphus punctipennis (Reuter) 227

Solidago 203, 204

-virgaurea L. 83, 86, 150, 203

Sorbus aucuparia 6

Sphagnum 2

Spiraea media Fr. Schmidt 203, 207

Stachys 219

- officinalis 72

- sylvatica 138

Stellaria 137

- holostea 148

Stenodema (Brachystira) calcarata (Fallén) 219

- (B.) pilosa (Jakovlev) 219

- (Stenodema) turanica Reuter 220

Sthenaropsis obscurus Poppius 225, 226

- piperatus Linnavuori 225

- schachrudicus Linnavuori 225, 226

Stigmus pendulus (Panzer) 247, 248

Stilpnus parvulus Foerster 110

- pavoniae (Scopoli) 110

- rectangulus Roman 110

- adanaensis Kolarov \& Beyarslan 109, 112

- blandus Gravenhorst 109

— bottnicus Jussila 108, 109

— crassicornis Thomson 109

- dryadum Curtis 107

- fallax (Foerster) 107, 108 
- gagates (Gravenhorst) 109

- Gravenhorst 107, 109, 110, 111

- montanus (Foerster) 108

- oligomerus (Foerster) 108

- subzonulus Foerster 110

- tenebricosus (Gravenhorst) 107

Stirophylus aristidae Wagner 226

Stomosema Fischer 130

Stratiotes aloides 68

Stylocryptus (Endasys) erythrogaster var. annulata 176

Stylops melittae Kirby 4

Suaeda 228

- dendroides 223, 228

- microphylla 227

Sympecma 127

Symphoricarpos 203

Symphoricarpus racemosus 83

- rivularis 82

Symphytum officinale 143

Syringa 203

— vulgaris L. 203

Syrphus admirandus Goeldlin 1

\section{$\mathbf{T}$}

Tachina sp. 71

Tamarix 229, 230

Tanacetum parthenium 77

Taraxacum 84

- offcinale 81, 133

Taylorilygus apicalis (Fieber) 221

Tenthredo 205

- rustica L. 203

- tricincta 202, 203

- vespa inaffectata Muche 202, 207

- vespa L.202, 203, 205, 206, 207

- vespa Retzius 201, 202, 205, 206

Teratocoris antennatus (Boheman) 219

Tetratoma fungorum Fabricius 3, 4

Teucrium polium 234

Thalictrum 138

- minus 134

Thaumatomyia Zenker 8, 9

- hallandica 19

- trifasciata 10, 19

- glabra (Meigen) 9, 12, 15, 19

- hallandica Andersson 9, 10, 12, 16

- notata (Meigen) 9, 10, 12, 19, 16

- rufa (Macquart) 9, 10, 16
- trifasciata (Zetterstedt) 9, 16

Therobolus Foerster 70

- ruficeps 72

Thymus 6, 222

Tingis (Neolasiotropis) pauperata (Puton) 234

- (N.) reuteri Horváth 234

- (Tingis) auriculata (Costa) 234

- (T.) leptochila Horváth 234

Tolbia Cameron 130

- scaevolae Cameron 130

Tomicus minor Hart. 31

Torilis japonica (Houtt.) 203

Tournefortia sogdiana 228

Trachyusa Ruthe 199, 200

- aurora (Haliday) 199, 200

- aurora ab. bicolor 200

- nigrothoracica 200

- nigrothoracica van Achterberg \& O'Connor 199

Tragopogon pratensis 132

Trichieurina Meigen 8

- pubescens (Meigen) 8, 10, 14

Trichonchus hackmani Millidge 6

Trifolium 86

— dubium 79

Trigonotylus caelestialium (Kirkaldy) 220

- pulchellus (Hahn) 220

- tenuis Reuter 220

Trilobomyza labiatarum Hendel 72, 138

- verbasci Bouché 72

Triticum 87, 88, 131

- sativum 94

Trypeta 77

- artemisiae Fabricius 77

_ immaculata (Macquart) 84, 146

Tuberculatus annulatus (Hartig) 248

Tuponia (Cholotuponia) kiritshenkoi

Drapolyuk 230

- (Chlorotuponia) concinna Reuter 230

Tuponia (Chlorotuponia) prasina (Fieber) 229

- (Tuponia) dehshorana Linnavuori 229

- (T.) ninlil Linnavuori 229

- (T.) algirica Wagner 229

- (T.) ayasensis Wagner 229

- (T.) elegans (Jakovlev) 229

- (T.) mixticolor (A. Costa) 229

- (T.) persica Wagner 229

- (T.) subaltera Drapolyuk 229

Tussilago farfara L. 83 
Typha latifolia 14

Tytthus parviceps (Reuter) 226

U

Ulmus 219, 222, 240

Urentius nanus (Schumacher) 234

Urtica 221

— dioica 84, 86, 87, 131, 138, 143

Utetes Foerster 65, 74

V

Verbascum 222, 224

- nigrum. 137

— phlomoides 137

- thapsiforme Schrad. 136, 137

- thapsus 137

Vespa biglumis Linnaeus, 1758193

- gallica Linnaeus, 1762194

Viburnum 203

Vicia faba 133

- sepium 74, 137

- sylvatica 147
- tenuifolia 88

- tetrasperma 79

Vidalia cornuta (Scopoli) 84

- spinifrons Schröder 134

Voruchia vittigera Reuter 227

Voruchiella haloxyli Puchkov 229

— pallida (Reuter) 229

- plagiata Poppius 229

- tincta (Jakovlev) 229

$\mathbf{X}$

Xestophyes Foerster 107, 110

Xynobius Foerster 68

Xynobius pallipes Foerster 68

$\mathbf{Z}$

Zeiraphera griseana (Hübner) 175

Zelotes electus (C.L. Koch) 6

- longipes (L.Koch) 6

- praeficus (L. Koch) 6

Zetetes 147

- ultor Foerster 149

Zizyphus sativa 144 